Research Paper

\title{
Benefits of surgery in the multimodality treatment of stage IIB-IIIC small cell lung cancer
}

\author{
Yong Yang1,2\#, Guangda Yuan 2,3\#, Cheng Zhan ${ }^{3 \#, ~ Y i w e i ~ H u a n g 33, ~ M e n g n a n ~ Z h a o ~} 3$, Xiaodong Yang3 , Shuai \\ Wang 3 , Zongwu Lin ${ }^{3}$, Shiying Zheng ${ }^{\circledR}$, Tao Lu ${ }^{3}$, Weigang Guo $^{3 凶}$, Qun Wang ${ }^{3}$ \\ 1. Department of Cardio-Thoracic Surgery, First Affiliated Hospital of Soochow University, Suzhou, Jiangsu Province, China \\ 2. Department of Thoracic Surgery, Suzhou Hospital affiliated to Nanjing Medical University, Suzhou, Jiangsu Province, China \\ 3. Department of Thoracic Surgery, Zhongshan Hospital, Fudan University, Shanghai, China \\ \#Yong Yang, Guangda Yuan and Cheng Zhan contributed equally to this work.
}

$\square$ Corresponding author: Shiying Zheng. Department of Thoracic Surgery, First Affiliated Hospital, Soochow University, No. 188, Shizi Road, Suzhou City, Jiangsu Province 215006, China. E-mail: drzhengshiying@126.com (Shiying Zheng). Tao Lu and Weigang Guo. Department of Thoracic Surgery, Zhongshan Hospital, Fudan University, 180 Fenglin Road, Shanghai, 200032, China. E-mail: lutao901118@163.com (Tao Lu); guo.weigang@zs-hospital.sh.cn (Weigang Guo)

() The author(s). This is an open access article distributed under the terms of the Creative Commons Attribution License (https://creativecommons.org/licenses/by/4.0/). See http://ivyspring.com/terms for full terms and conditions.

Received: 2018.11.03; Accepted: 2019.07.28; Published: 2019.08.29

\begin{abstract}
Surgery combined with chemotherapy/radiotherapy is recommended for early stage small cell lung cancer (SCLC); however, the role of surgery in the multimodality treatment of advanced disease remains controversial. The clinical data of patients between 2000 and 2015 were obtained from the Surveillance, Epidemiology, and End Results database. The surgery group included 998 patients with stage IIB-IIIC. A matched non-surgery group $(n=2994)$ was generated by propensity score matching. The Kaplan-Meier method and log-rank tests were used for survival analyses. Univariate and multivariate analyses were used to identify significant prognostic factors. After matching, there were no significant differences between the two groups in race, age, sex, $T$ classification, $N$ classification, TNM stage, marital status, primary sites, and origin record NAACCR Hispanic Identification Algorithm (NHIA). The surgery group showed better overall survival and cancer-specific survival than the non-surgery group. Univariate and multivariate analyses showed that therapy methods, age, sex, $\mathrm{T}$ classification, and $\mathrm{N}$ classification were independent prognostic predictors for stage IIB-IIIC SCLC (all $P<0.05$ ). Stratified analyses showed that survival outcomes favored surgery in any age groups, men and women, any T classification except T3, and N0-2 but not N3 compared with non-surgical treatment. The survival differences favored surgery in stage IIB and IIIA SCLC, although they were not significant in stage IIB and IIIC SCLC. Therefore, surgery was associated with improved survival in stage IIB and IIIA SCLC, but not in stage IIIB and IIIC SCLC.
\end{abstract}

Key words: small cell lung cancer, surgery, non-surgery, propensity score matching, survival

\section{Introduction}

Small cell lung cancer (SCLC) accounts for approximately $15 \%$ of new lung cancer cases annually. It is a virulent, rapidly growing, early metastasizing, and highly invasive cancer with a 5 -year survival rate of $<7 \% 1,2$. Most of patients with SCLC already have regional or distant spread at diagnosis ${ }^{3}$. Despite recent advances in the treatment of SCLC, patient survival only shows a slight improvement ${ }^{4}$.
Currently, chemotherapy and radiotherapy are the standard treatments for patients with SCLC, and they have provided patients a chance to prolong their survival ${ }^{5}$. Surgical treatment is only considered to be limited as an option for early stage SCLC according to recent consensus. The NCCN guidelines version 2 (2018) suggest that patients with SCLC at a stage higher than T1-2 with N0 do not benefit from surgery 6. However, reports show that surgery can 
significantly improve survival outcomes in SCLC patients and should be considered in the management of SCLC at stages higher than T1-2 with N0 7 .

The role of surgery in the multimodality treatment of advanced SCLC remains controversial. Here, we investigated whether patients with stage IIB-IIIC SCLC could benefit from surgical resection as part of the multimodality treatment based on chemotherapy/radiotherapy.

\section{Material and Methods}

\section{Data acquisition}

The data of patients with small lung cancer were obtained from the Surveillance, Epidemiology, and End Results (SEER) database (Incidence-SEER 18 Regs Custom Data with additional treatment fields, Nov 2017 Sub, 1973 - 2015 varying) via SEER*Stat, version 8.3 .5 (http:// seer. cancer.gov/ seerstat/).

The data of patients with SCLC are shown in Figure 1A. Briefly, patients with SCLC (ICD-O-3 $8041 / 3,8043 / 3,8044 / 3,8045 / 3$ ) were recruited between 2000 and 2015. The labeled primary sites were limited to C34.1-upper lobe, lung, C34.2-middle lobe, lung, C34.3-lower lobe, lung, and C34.8-Overlapping lesion of lung, C34.9-lung, NOS. The old version of tumor TNM stage was converted to the new AJCC TNM stage manually. The inclusion criteria were as follows: (1) Patients with stage IIB-IIIC disease; and (2) patients treated with chemotherapy or radiotherapy or both. The exclusion criteria were as follows: (1) Patients diagnosed with stage I, IIA, and IV SCLC; (2) patients who did not receive chemotherapy/radiotherapy or surgery; (3) patients who underwent surgical treatment alone. After selection, all patients enrolled in the study were divided into a surgery group and a non-surgery (chemotherapy/radiotherapy) group according to the mode of therapy.

The data of patient and tumor characteristics, including age, sex, race, year of diagnosis, marital status at the time of diagnosis, origin recode NAACCR Hispanic Identification Algorithm (NHIA), primary site, $\mathrm{T}$ classification, $\mathrm{N}$ classification, TNM stage, surgery recode, chemotherapy recode, radiation recode, survival time, and survival outcomes were derived from the database. Age was categorized as $\leq 60$ years, $6-70$ years, and $>70$ years.

\section{Study design}

The propensity score matching (PSM) method was used to overcome patient selection bias. The following factors were matched between the two groups: age, sex, race, primary sites, $\mathrm{T}$ classification, $\mathrm{N}$ classification, TNM stage, marital status at the time of diagnosis, and origin recode NHIA. For each case in the surgery group, three cases in non-surgery group were randomly chosen for pairing by PSM. The non-SCLC cancer-specific survival outcomes were used to perform competing risk analyses.

Statistical analyses were performed using IBM SPSS Statistics 24.0 (IBM, Inc., Armonk, NY) and R version 3.5.1 (R Foundation for Statistical Computing, Vienna, Austria). Before and after matching, the characteristics between the two groups were compared using chi-square tests. A standardized difference less than $10 \%$ was acceptable to evaluate the balance of covariates before and after matching. The overall survival (OS) and the cause-specific survival (CSS) were estimated by the Kaplan-Meier method, and log-rank tests were used to analyze differences between curves. Univariate and multivariate Cox regression analyses were used to identify significant prognostic factors. Two-sided $P$ values $<0.05$ were considered statistically significant.

\section{Ethics statements}

Ethics approval was exempted by the Ethics Committee of Zhongshan Hospital of Fudan University (Shanghai, China), as the SEER is a publicly available database, and data extracted from SEER were identified as nonhuman study.

\section{Results}

\section{Patients characteristics}

There were 85462 patients with SCLC in the SEER database between 2000 and 2015. After rigorous selection, 998 patients with stage IIB-IIIC SCLC who underwent surgery combined with chemotherapy or radiotherapy or both were included in the surgery group, and 17467 patients who only received chemotherapy or radiotherapy or both were included in the non-surgery group. After matching based on propensity scores, 2994 patients were selected and enrolled in the non-surgery group (Figure 1A). As shown in Table 1, compared to the patients who received non-surgical treatment, the patients who received surgery were more likely to be white and married, were more easily to develop tumors located in lower lobe, and had a higher tumor T classification, $\mathrm{N}$ classification and TNM stage (all $P<0.05$ ). After matching, there were no significant differences between the two groups in terms of age, sex, race, origin record NHIA, marital status, primary sites, TNM stage, $\mathrm{T}$ classification, and $\mathrm{N}$ classification (all $P$ $>0.05$; Figure 1B).

\section{Survival analyses}

The 1, 2, and 3-year OS rates and CSS rates of patents with stage IIB-IIIC SCLC in the two groups were shown in Table 2. Generally, patients in the 
surgery group had better OS and CSS rates than those in the non-surgery group $(P<0.001$; Figure $2 \mathrm{~A}$ and $\mathrm{B})$. Competing risk model analysis showed that the patients who received non-surgical treatment had a higher risk of cause-specific death from SCLC $(P<$ 0.001 ), and there was no significant difference in the probabilities of other causes of death $(P=0.777$; Figure 2C).

Table 1: Patient characteristics before and after matching

\begin{tabular}{|c|c|c|c|c|c|c|}
\hline \multirow[t]{2}{*}{ Characteristics } & \multicolumn{3}{|c|}{ Before matching } & \multicolumn{3}{|c|}{ After matching } \\
\hline & Non-surgery & Surgery & $P$ & Non-surgery & Surgery & $P$ \\
\hline Age (mean) & 66.26 & 66.74 & 0.131 & 67.22 & 66.74 & 0.162 \\
\hline Age group & & & 0.019 & & & 0.753 \\
\hline$\leq 60$ & 4896 & 239 & & 702 & 239 & \\
\hline $60-70$ & 6363 & 389 & & 1142 & 389 & \\
\hline$>70$ & 6208 & 370 & & 1150 & 370 & \\
\hline Sex & & & 0.441 & & & 0.464 \\
\hline Male & 8252 & 459 & & 1417 & 459 & \\
\hline Female & 9215 & 539 & & 1577 & 539 & \\
\hline Race & & & 0.001 & & & 0.757 \\
\hline White & 15075 & 902 & & 2723 & 902 & \\
\hline Black & 1649 & 69 & & 202 & 69 & \\
\hline Others & 743 & 27 & & 69 & 27 & \\
\hline NHIA & & & 0.08 & & & 0.165 \\
\hline SHL & 650 & 48 & & 114 & 48 & \\
\hline NSHL & 16817 & 950 & & 2880 & 950 & \\
\hline Tumor Site & & & $<0.001$ & & & 0.866 \\
\hline Upper lobe & 9928 & 572 & & 1726 & 572 & \\
\hline Middle lobe & 829 & 55 & & 141 & 55 & \\
\hline Lower lobe & 3786 & 308 & & 942 & 308 & \\
\hline Overlapping & 281 & 22 & & 70 & 22 & \\
\hline Lung, NOS & 2643 & 41 & & 115 & 41 & \\
\hline Marital status & & & 0.001 & & & 0.815 \\
\hline Married & 9014 & 572 & & 1710 & 572 & \\
\hline Ever-married & 5820 & 306 & & 942 & 306 & \\
\hline Never-married & 1985 & 81 & & 243 & 81 & \\
\hline Unknown & 648 & 39 & & 99 & 39 & \\
\hline
\end{tabular}

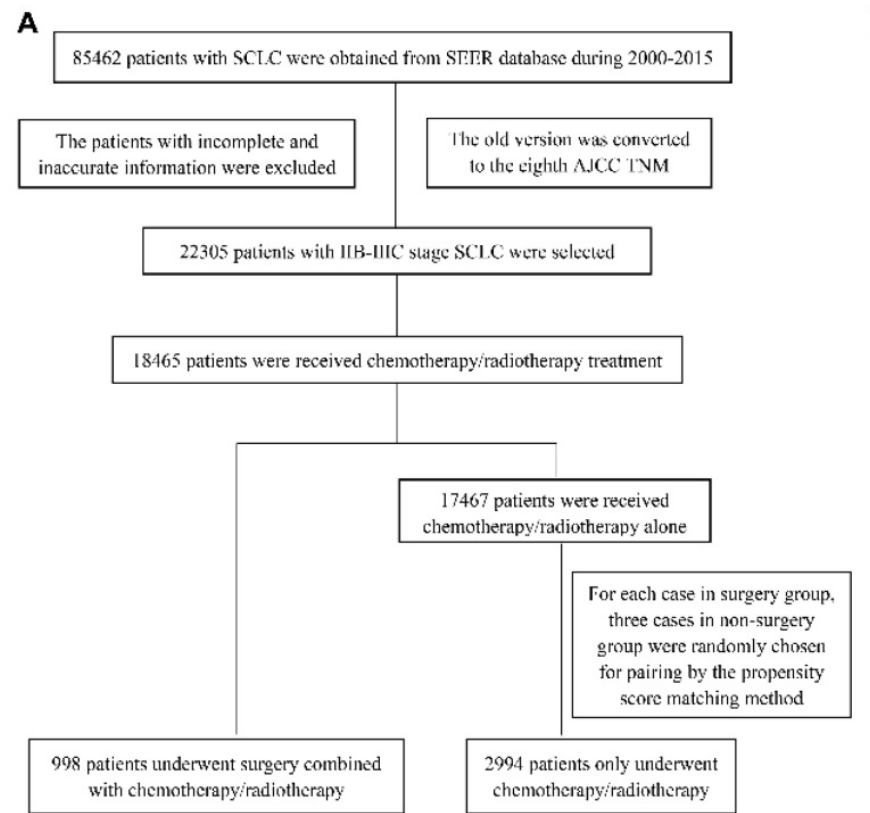

\begin{tabular}{|c|c|c|c|c|c|c|}
\hline \multirow[t]{2}{*}{ Characteristics } & \multicolumn{3}{|c|}{ Before matching } & \multicolumn{3}{|c|}{ After matching } \\
\hline & Non-surgery & Surgery & $P$ & Non-surgery & Surgery & $P$ \\
\hline TNM stage & & & $<0.001$ & & & 1.000 \\
\hline IIB & 1284 & 388 & & 1164 & 388 & \\
\hline IIIA & 6667 & 470 & & 1410 & 470 & \\
\hline IIIB & 7747 & 131 & & 393 & 131 & \\
\hline IIIC & 1769 & 9 & & 27 & 9 & \\
\hline $\mathrm{T}$ classification & & & $<0.001$ & & & 0.934 \\
\hline T1 & 4189 & 362 & & 1090 & 362 & \\
\hline $\mathrm{T} 2$ & 2282 & 241 & & 723 & 241 & \\
\hline $\mathrm{T} 3$ & 2122 & 179 & & 513 & 179 & \\
\hline $\mathrm{T} 4$ & 8874 & 216 & & 668 & 216 & \\
\hline $\mathrm{N}$ classification & & & $<0.001$ & & & 0.235 \\
\hline No & 1745 & 188 & & 626 & 188 & \\
\hline $\mathrm{N} 1$ & 1600 & 363 & & 991 & 363 & \\
\hline N2 & 11354 & 422 & & 1295 & 422 & \\
\hline N3 & 2768 & 25 & & 82 & 25 & \\
\hline
\end{tabular}

NHIA: NAACCR Hispanic Identification Algorithm

NSHL: Non-Spanish-Hispanic-Latino

SHL: Spanish-Hispanic-Latino

The correlation between survival and other parameters was further investigated. Univariate analyses showed that age $(P<0.001)$, sex $(P<0.001)$, marital status $(P=0.009)$, TNM stage $(P<0.001)$, T classification $(P<0.001)$, N classification $(P<0.001)$, and therapy methods $(P<0.001)$ were statistically significant predictors of OS (Table 3). There was no significance in terms of race $(P=0.131)$, origin record NHIA $(P=0.941)$, and tumor primary site $(P=0.763)$. Based on multivariate analyses, age $(P<0.001)$, sex $(P$ $<0.001)$, T classification $(P<0.001)$, $\mathrm{N}$ classification $(P$ $=0.004)$, and therapy methods $(P<0.001)$ were independent prognostic predictors for stage IIB-IIIC SCLC.

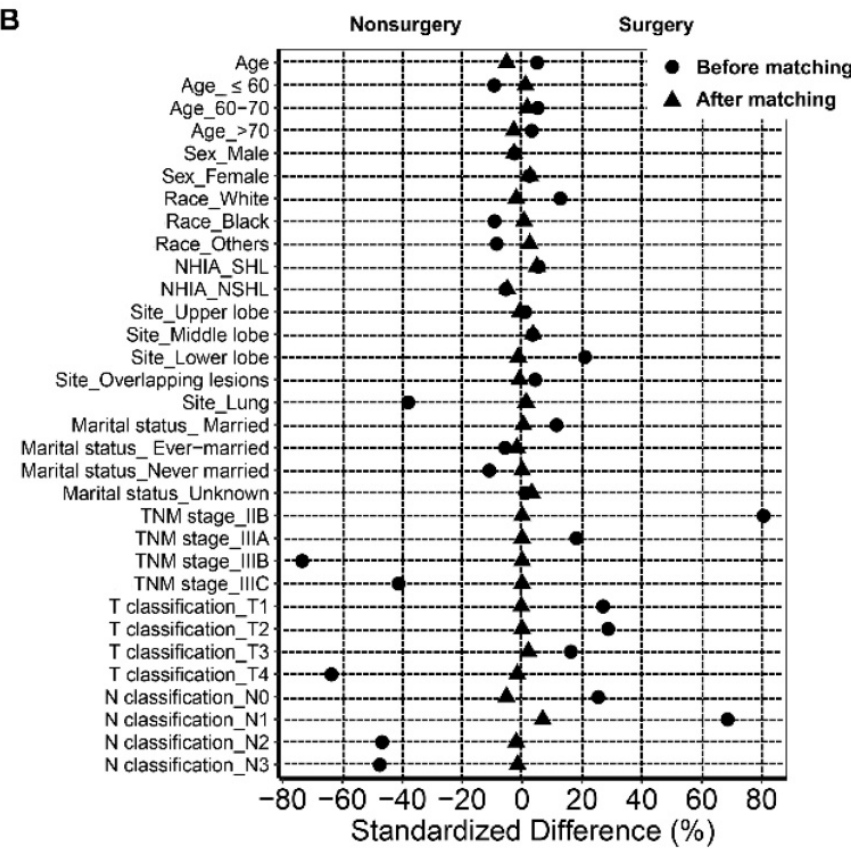

Figure 1. Flow chart of the selection of cases and controls (A); Standardized differences of baseline variables before and after propensity score matching (B). 
Table 2: The survival rate in non-surgery group and surgery group

\begin{tabular}{llcl}
\hline Survival rate & Total & Non-surgery group & Surgery group \\
\hline 1-year OS & $62.4 \%$ & $60.6 \%$ & $67.8 \%$ \\
1-year CSS & $66.9 \%$ & $65.4 \%$ & $71.5 \%$ \\
2-year OS & $36.7 \%$ & $35.1 \%$ & $41.5 \%$ \\
2-year CSS & $42.1 \%$ & $40.4 \%$ & $46.8 \%$ \\
3-year OS & $26.7 \%$ & $24.8 \%$ & $32.6 \%$ \\
3-year CSS & $32.2 \%$ & $30.1 \%$ & $38.0 \%$ \\
\hline
\end{tabular}

OS: overall survival

CSS: cause-specific survival

Table 3: Univariate and multivariate analyses of overall survival of all patients after matching

\begin{tabular}{lllllll}
\hline Variables & \multicolumn{3}{c}{ Univariate Analysis } & \multicolumn{4}{c}{ Multivariate analysis } \\
\cline { 2 - 7 } & HR & $95 \%$ CI & $P$ & HR & $95 \%$ CI & $P$ \\
\hline Age & & & $<0.001$ & & & $<0.001$ \\
$\leq 60$ & Reference & Reference & & Reference & Reference & \\
$60-70$ & 1.370 & $1.247-1.506$ & $<0.001$ & 1.369 & $1.245-1.506$ & $<0.001$ \\
$>70$ & 1.850 & $1.684-2.033$ & $<0.001$ & 1.865 & $1.694-2.053<0.001$ \\
Sex & & & & & & \\
Male & Reference & Reference & & Reference & Reference & \\
Female & 0.835 & $0.779-0.896$ & $<0.001$ & 0.818 & $0.760-0.880$ & $<0.001$ \\
Race & & & 0.131 & & & \\
White & Reference & Reference & & & & \\
Black & 0.896 & $0.778-1.031$ & 0.126 & & & \\
Others & 0.849 & $0.672-1.072$ & 0.169 & & & \\
NHIA & & & & & & \\
SHL & Reference & Reference & & & & \\
NSHL & 0.933 & $0.831-1.187$ & 0.941 & & & \\
Tumor Site & & & 0.763 & & & \\
Upper lobe & Reference & Reference & & & & \\
Middle lobe & 1.017 & $0.866-1.195$ & 0.835 & & & \\
Lower lobe & 1.044 & $0.962-1.132$ & 0.302 & & & \\
Overlapping & 1.107 & $0.829-1.479$ & 0.491 & & & \\
Lung, NOS & 0.975 & $0.859-1.106$ & 0.689 & & & \\
Marital status & & & 0.009 & & & \\
Married & Reference & Reference & & Reference & Reference & \\
Ever-married & 1.107 & $1.025-1.196$ & 0.010 & 1.105 & $1.019-1.200$ & 0.016 \\
\hline
\end{tabular}

\section{A}

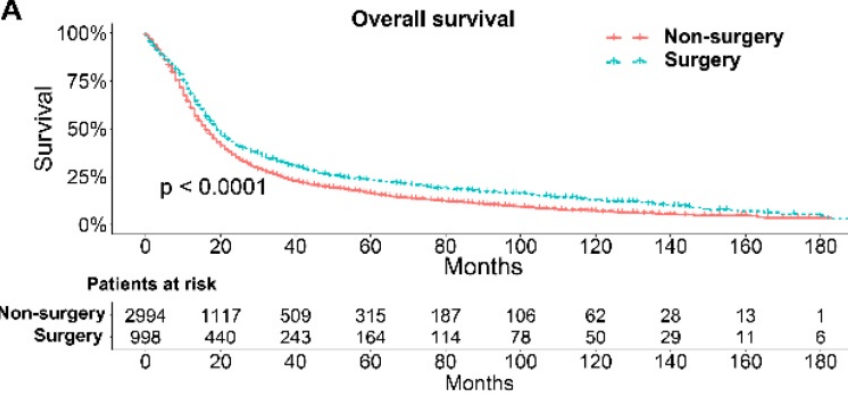

B

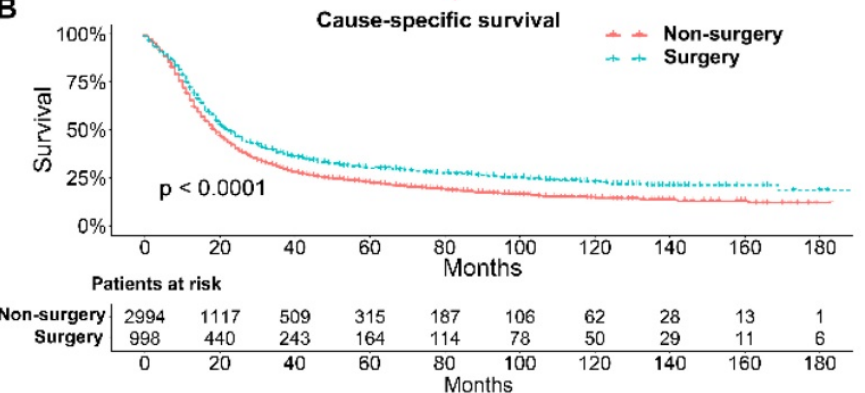

\begin{tabular}{|c|c|c|c|c|c|c|}
\hline \multirow[t]{2}{*}{ Variables } & \multicolumn{2}{|c|}{ Univariate Analysis } & \multicolumn{4}{|c|}{ Multivariate analysis } \\
\hline & HR & $95 \% \mathrm{CI}$ & $P$ & HR & $95 \% \mathrm{CI}$ & $P$ \\
\hline Never-married & 0.894 & $0.780-1.024$ & 0.105 & 1.028 & $0.896-1.179$ & 0.698 \\
\hline Unknown & 0.985 & $0.808-1.202$ & 0.885 & 1.079 & $0.884-1.317$ & 0.454 \\
\hline TNM stage & & & $<0.001$ & & & \\
\hline IIB & Reference & Reference & & & & \\
\hline IIIA & 1.206 & $1.117-1.302$ & $<0.001$ & & & \\
\hline IIIB & 1.540 & $1.380-1.717$ & $<0.001$ & & & \\
\hline IIIC & 1.898 & $1.321-2.728$ & $<0.001$ & & & \\
\hline $\mathrm{T}$ class & & & $<0.001$ & & & $<0.001$ \\
\hline $\mathrm{T} 1$ & Reference & Reference & & Reference & Reference & \\
\hline $\mathrm{T} 2$ & 1.135 & $1.036-1.244$ & 0.007 & 1.112 & $1.015-1.219$ & 0.023 \\
\hline $\mathrm{T} 3$ & 0.946 & $0.851-1.051$ & 0.299 & 1.134 & $0.986-1.291$ & 0.058 \\
\hline $\mathrm{T} 4$ & 1.227 & $1.119-1.346$ & $<0.001$ & 1.360 & $1.226-1.509$ & $<0.001$ \\
\hline$N$ class & & & $<0.001$ & & & $<0.001$ \\
\hline No & Reference & Reference & & Reference & Reference & \\
\hline N1 & 1.027 & $0.930-1.135$ & 0.596 & 1.207 & $1.064-1.370$ & 0.004 \\
\hline $\mathrm{N} 2$ & 1.245 & & $<0.001$ & 1.461 & $1.295-1.647$ & $<0.001$ \\
\hline N3 & 1.966 & $1.582-2.444$ & $<0.001$ & 2.134 & $1.697-2.685$ & $<0.001$ \\
\hline \multicolumn{7}{|l|}{ Therapy } \\
\hline Non-surgery & Reference & Reference & & Reference & Reference & \\
\hline Surgery & 0.809 & $0.746-0.878$ & $<0.001$ & 0.807 & $0.744-0.876$ & $<0.001$ \\
\hline
\end{tabular}

To further explore the influence of therapy methods on the survival of stage IIB-IIIC SCLC patients, we stratified the matched patients by significant variables based on multivariate regression model analyses. In patients aged $\leq 60,60-70$, and $>70$ years, the survival rates were significantly better in the surgery group than in the non-surgery group (all $\mathrm{P}<0.05$; Figure 3A-C). Moreover, both male and female patients in the surgery group had better survival than those in the non-surgery group (all $P<$ 0.001; Figure 4A and B). In the analysis of tumor $\mathrm{T}$ classification, only patients with T3 SCLC had comparable survival outcomes between the surgery group and the non-surgery group $(P=0.28)$, whereas there were significant differences in T1 $(P=0.0014)$,

C

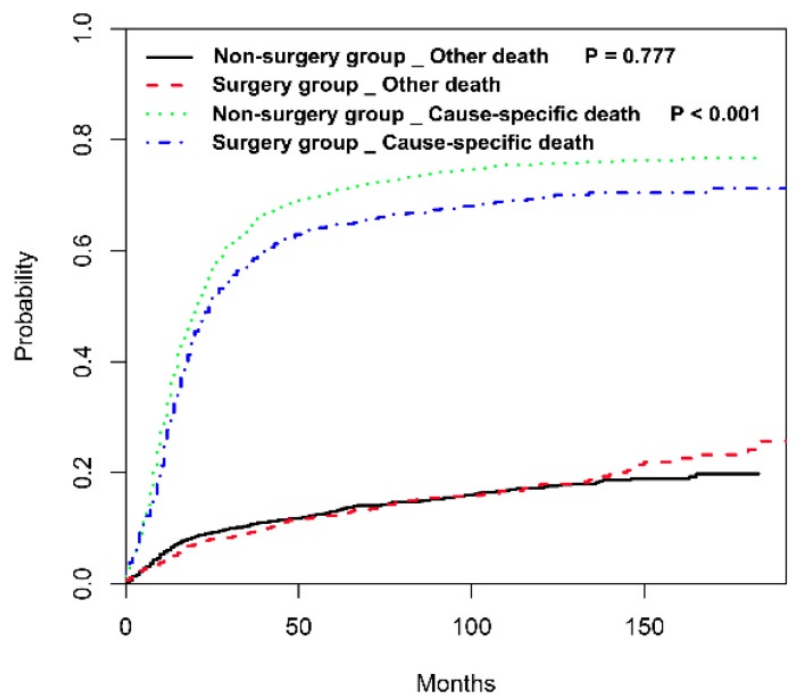

Figure 2. Survival analyses of patients with stage IIB-IIIC SCLC undergoing surgical treatment and non-surgical treatment. (A) Kaplan-Meier analysis of overall survival; (B) Kaplan-Meier analysis of cause-specific survival; (C) Competing risk analysis. 
T2 $(P=0.036)$, and T4 $(P<0.001)$ SCLC (Figure 5A-D). With respect to the $\mathrm{N}$ classification, surgical treatment was associated with better survival than non-surgical treatment in N0 $(P=0.0064), \mathrm{N} 1(P=0.001)$, and N2 $(P$ $=0.0022)$ SCLC, but not in N3 patients $(P=0.68$; Figure 6A-D). For tumor TNM stage, a survival advantage related to surgery was observed in patients with stage IIB $(P=0.003)$ and IIIA $(P<0.001)$ SCLC (Figure 7A and B). However, no significant difference in survival was observed in patients with stage IIIB ( $P$ $=0.23)$ and IIIC $(P=0.16)$ SCLC (Figure 7C and D).
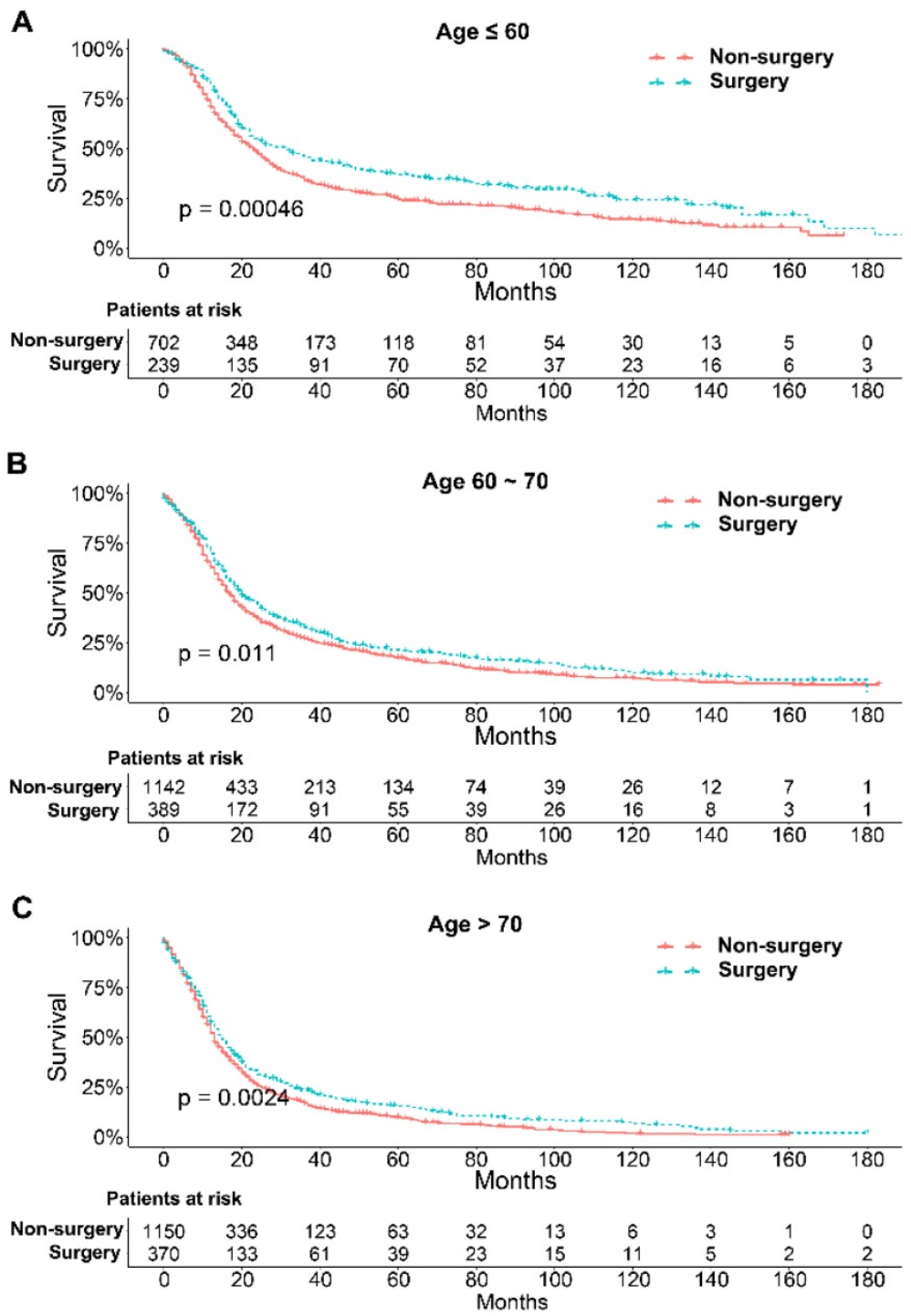

Figure 3. Survival analyses of patients undergoing surgical treatment and non-surgical treatment stratified by age after matching. (A) $\leq 60$ years of age; (B) $60-70$ years of age; (C) $>70$ years of age.
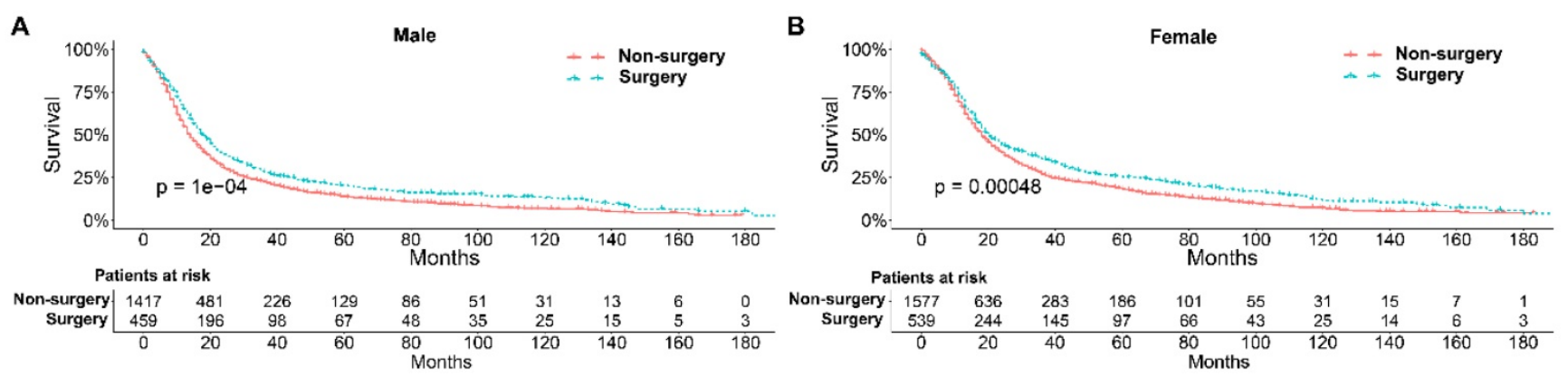

Figure 4. Survival analyses of patients undergoing surgical treatment and non-surgical treatment stratified by sex after matching. (A) male patients; (B) female patients. 

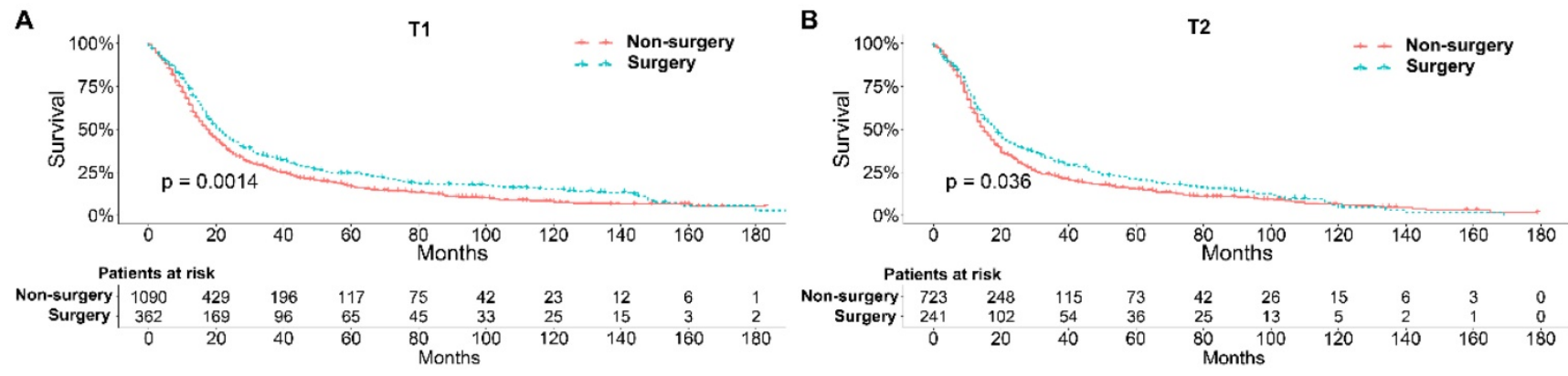

C

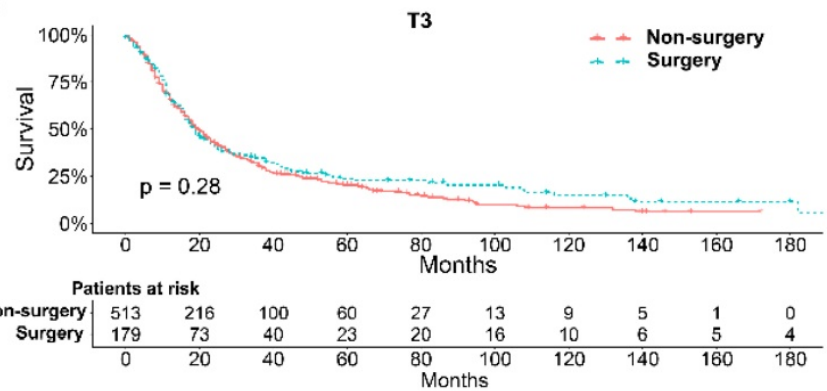

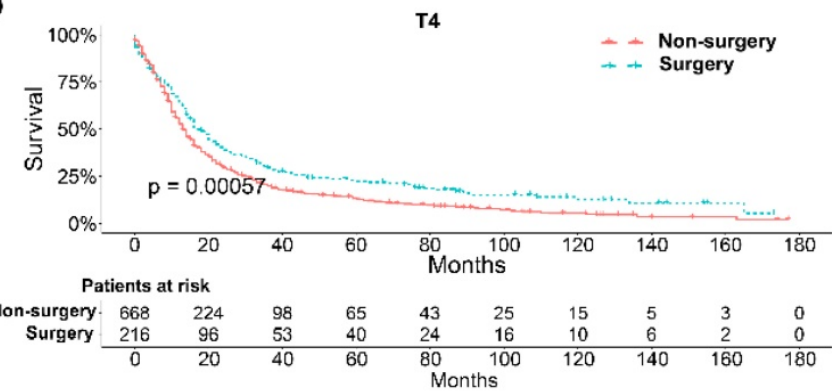

Figure 5. Survival analyses of patients undergoing surgical treatment and non-surgical treatment stratified by tumor T classification after matching. (A) T1 classification; (B) T2 classification; (C) T3 classification; (D) T4 classification.
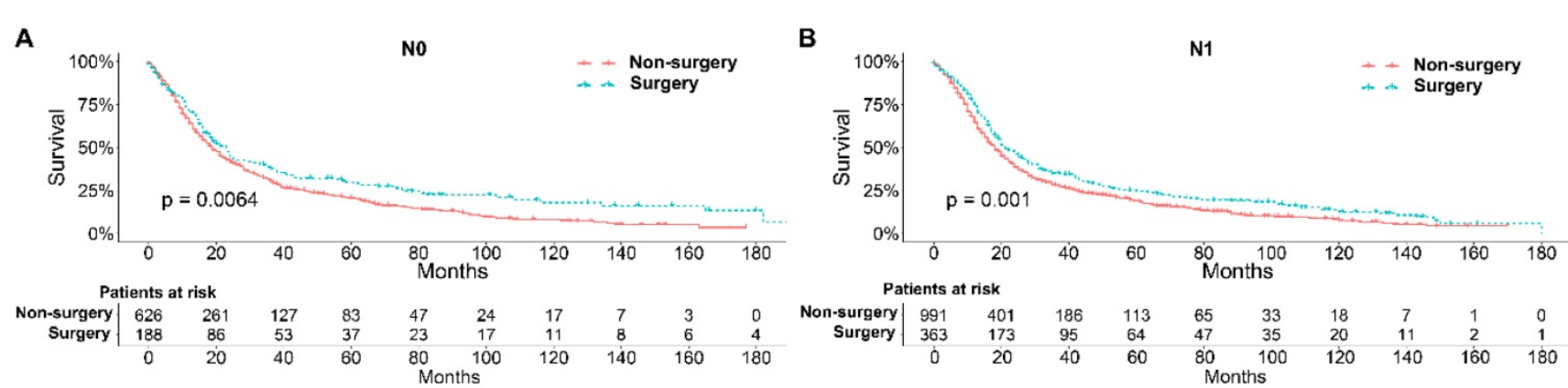

C

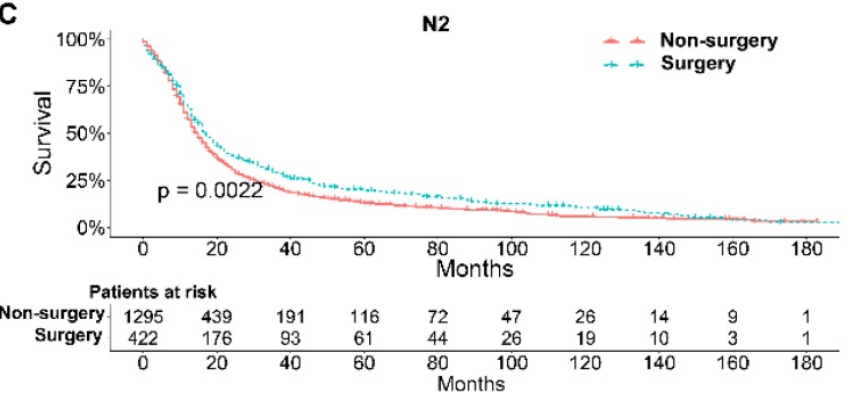

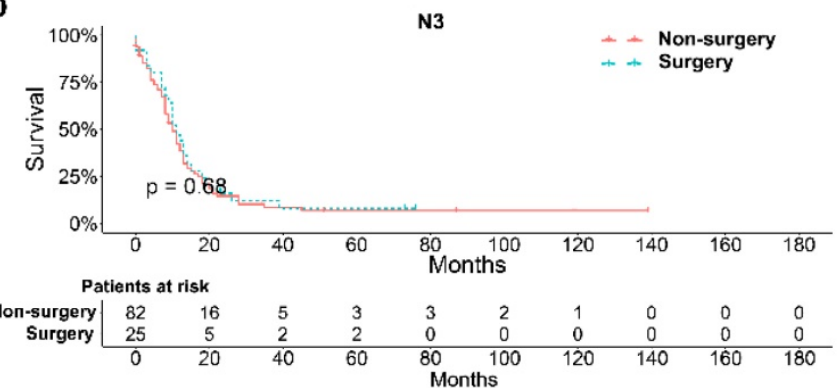

Figure 6. Survival analyses of patients undergoing surgical treatment and non-surgical treatment stratified by tumor N classification after matching. (A) N0 classification; (B) N1 classification; (C) N2 classification; (D) N3 classification.

\section{Discussion}

Previously, a large of studies including prospective and retrospective analyses focused on the treatment for NSCLC $8-15$, because NSCLC accounted for $85 \%$ of lung cancer cases ${ }^{16}$. It has been reported that surgery combined with chemotherapy/ radiotherapy may improve the survival of the patients with advanced NSCLC 9,15. For SCLC, the standard of care is chemotherapy and radiotherapy, even for limited disease ${ }^{17}$. The role of surgery in the treatment of advanced SCLC is controversy ${ }^{18-20}$. Several studies examined the role of surgery in the SCLC patients ${ }^{21-23,}$ but few studies solved the problem of potential selection biases in the analyses. In the present study, we used the PSM method to eliminate the selection bias by matching factors between the two groups, including age, sex, race, primary sites, $\mathrm{T}$ classification, $\mathrm{N}$ classification, TNM stage, marital status at the time of diagnosis, and origin record NIHA, and investigated the role of surgery in the chemotherapy/radiotherapy-based multimodality 
A

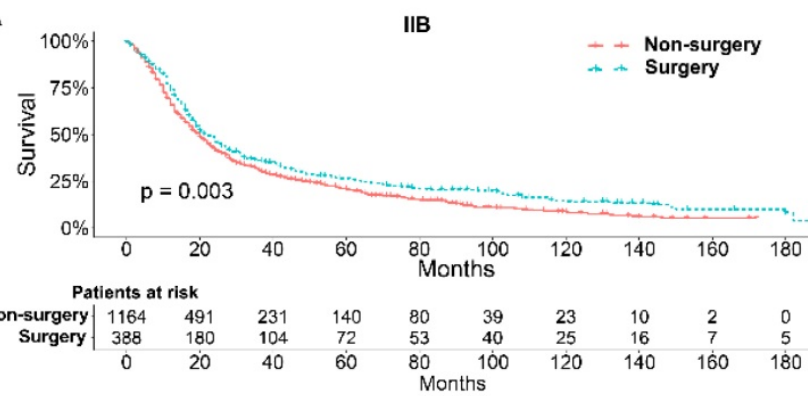

C

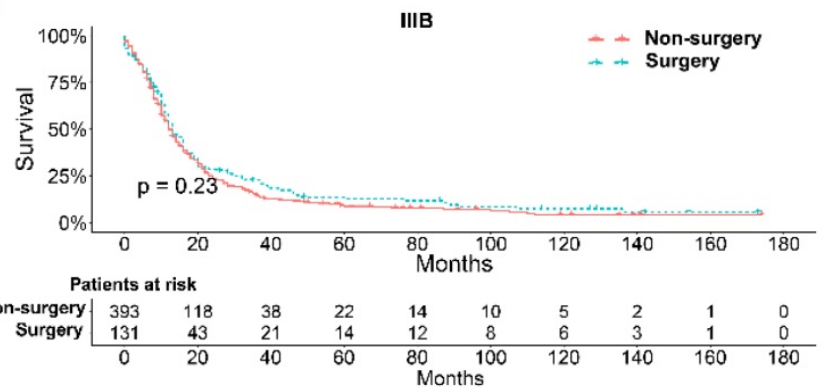

B

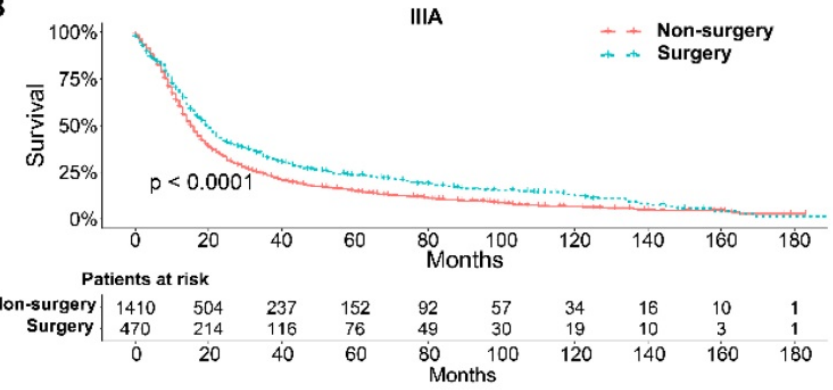

D

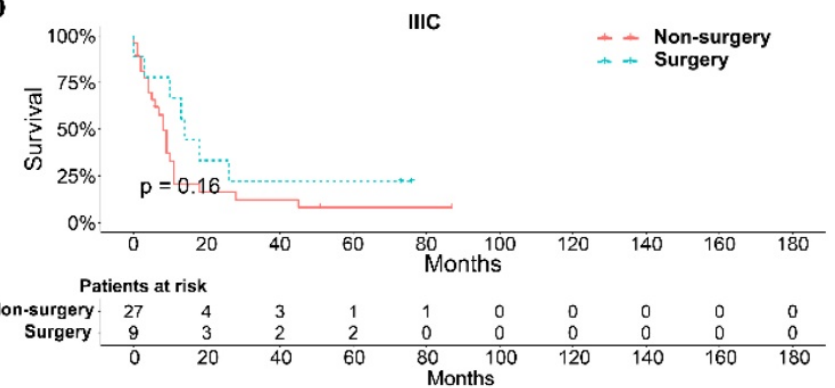

Figure 7. Survival analyses of patients undergoing surgical treatment and non-surgical treatment stratified by TNM stage after matching. (A) Stage IIB; (B) Stage IIIA; (C) Stage IIIB; (D) Stage IIIC.

treatment of patients with stage IIB-IIIC SCLC. To our knowledge, it is the first time to use PSM method to eliminate the patient selection bias among the baseline variables of the two groups to investigate the role of surgery in the treatment for advanced SCLC. In addition, we used the competing risk model to distinguish cancer-specific death from death from other causes. No significant difference was found in the probabilities of other causes of death. These processes resulted in a more accurate comparison of survival outcomes between the two groups.

SCLC is a disease with a grave prognosis. In the present study, the 1,2, and 3-year OS rates of patients with stage IIB-IIIC SCLC were $62.4 \%, 36.7 \% \%$, and $26.7 \%$, respectively, which was consistent with the results of the study by Eberhardt et al. ${ }^{24}$. Although surgery offers lung cancer patients the best chance of long-term survival, surgery alone is not sufficient, even for early stage SCLC. Analysis of the National Cancer DataBase indicated that patients with early SCLC who received surgery alone had a lower survival than those who received surgery combined with chemotherapy ${ }^{25}$. A retrospective review by Szczesny et al. showed that surgery alone was associated with lower 5-year survival rates than a multidisciplinary approach for very limited disease 20 . Furthermore, reports show that chemotherapy/ radiotherapy may be effective and could decrease the risk of recurrence in SCLC, and could provide a survival benefit to patients 26, 27. However, chemotherapy/radiotherapy alone is associated with poor prognosis when compared with surgery with adjuvant therapy for early stage SCLC 28, 29. These results support an increased role of surgery in the multimodality treatment of SCLC, and the combination of surgery with chemotherapy/ radiotherapy could be beneficial in patients with early stage SCLC. Increasing evidence indicates that multimodality treatment including surgical resection is associated with better survival than chemotherapy/radiotherapy alone 18, 30, 31. The treatment, namely surgery combined with adjuvant chemotherapy/radiotherapy, has a 5-year survival of approximately $40 \%-70 \%$ for limited-stage SCLC patients 26,32 .

In the past, surgery was only recommended for early stage SCLC 18 , as it has little beneficial effects in patients with advanced disease. However, studies favor surgical intervention in patients with stage III SCLC. Shepherd et al. ${ }^{33}$ showed that stage II (T1N1, T2N1) and stage III (any T3 or T1-2N2) SCLC patients who underwent surgery and adjuvant chemotherapy had a median survival of 72 weeks and 65 weeks, and projected 5-year survival rates of $24.5 \%$ and $24 \%$, respectively. Eberhardt et al. 34 showed that the addition of surgery to multimodality treatment provided significant long-term survival, even in locally advanced patients with stage IIB/IIIA SCLC. Tsuchiya et al. ${ }^{32}$ and Combs et al. ${ }^{23}$ also reported comparable results. In the present study, OS and CSS were better in patients with stage IIB-IIIC SCLC in the surgery group than in those in non-surgery group, indicating that the inclusion of surgery in the multimodality treatment is beneficial for longer survival. 
Survival differences stratified by significant variables were analyzed to investigate the impact of therapeutic approaches on the survival of matched patients receiving surgical treatment and non-surgical treatment. The results of multivariate Cox proportional hazards analyses indicated that age, sex, T classification, $\mathrm{N}$ classification, and therapy methods were significant independent predictors of survival outcome. Generally, elderly patients with SCLC had worse survival probably because they have a significantly higher number of comorbidities, smaller bone marrow reserve, and poor performance status that may affect the tolerance of chemotherapy 35,36 . Our results indicated that surgical treatment could be beneficial for patients with stage IIB-IIIC SCLC of all ages compared with non-surgical treatment. With respect to sex, men had higher smoking rates than women at any time point, which may contribute to the higher mortality rate ${ }^{37}$. We showed that surgical treatment was associated with significantly better survival than non-surgical treatment for both male and female patients. Matched Kaplan-Meier survival analyses showed that surgery was associated with longer survival for $\mathrm{T}$ and $\mathrm{N}$ classification SCLC, although the difference was not significant for T3 $(P=$ $0.28)$ and N3 $(P=0.68)$ classifications. The results were consistent with those of a study performed by Wakeam et al. ${ }^{38}$. They showed that the survival differences favored surgery in any $\mathrm{T}$ classification and both N1 and N2 positive cohorts compared with non-surgical treatment, which indicated that surgery plays an important role in the multimodality treatment of SCLC. In our study, we also did a stratified analysis of survival by TNM stage between the two groups. Consistent with a previous study 38 , we showed that surgery for stage IIB and IIIA SCLC was associated with improved long-term survival compared with nonsurgical treatment. Moreover, compared with previous studies, we also showed that surgery in the multimodality treatment had no influence on the survival of patients with stage IIIB and IIIC SCLC.

\section{Limitations}

The present study had several limitations. First, the information available in the SEER database regarding chemotherapy and radiotherapy for SCLC was limited, and we were unable to obtain specific information regarding chemotherapy drugs, radiotherapy dose, and the treatment course. Second, the tumor differentiation in most cases in the non-surgery group was unknown, and the impact of tumor differentiation on survival outcomes could not be analyzed. Third, certain patients were excluded because of incomplete or inaccurate clinical information, which may lead to potential selection bias.

\section{Conclusions}

The present study demonstrated that the combination of surgery with chemotherapy/ radiotherapy was associated with better survival outcomes than conventional chemotherapy/ radiotherapy alone in stage IIB and IIIA SCLC, but not in stage IIIB and IIIC SCLC. However, surgical resection was not associated with improved survival outcomes in the management of T3 classification and N3 classification.

\section{Abbreviations}

SCLC: small cell lung cancer; SEER: Surveillance, Epidemiology, and End Results; NHIA: NAACCR Hispanic Identification Algorithm; NSHL: Non-Spanish-Hispanic-Latino; SHL: SpanishHispanic-Latino; PSM: propensity score matching; OS: overall survival; CSS: cause-specific survival.

\section{Acknowledgments}

We thank International Science Editing (http://www.internationalscienceediting.com) for editing this manuscript.

\section{Funding}

This work was supported by the National Natural Science Foundation of China (Grant No. 81572295), and the Shanghai Sailing Program (No.17YF1402400).

\section{Competing Interests}

The authors have declared that no competing interest exists.

\section{References}

1. Xie D, Marks R, Zhang M, Jiang G, Jatoi A, Garces YI. et al. Nomograms Predict Overall Survival for Patients with Small-Cell Lung Cancer Incorporating Pretreatment Peripheral Blood Markers. J Thorac Oncol. 2015;10:1213-20.

2. Jett JR, Schild SE, Kesler KA, Kalemkerian GP. Treatment of small cell lung cancer: Diagnosis and management of lung cancer, 3rd ed: American College of Chest Physicians evidence-based clinical practice guidelines. Chest. 2013;143:e400S-e19S

3. Sher T, Dy GK, Adjei AA. Small cell lung cancer. Mayo Clin Proc. 2008;83:355-67.

4. Govindan R, Page N, Morgensztern D, Read W, Tierney R, Vlahiotis A. et al. Changing epidemiology of small-cell lung cancer in the United States over the last 30 years: analysis of the surveillance, epidemiologic, and end results database. J Clin Oncol. 2006;24:4539-44.

5. Buchholz E, Manegold C, Pilz L, Thatcher N, Drings P. Standard versus dose-intensified chemotherapy with sequential reinfusion of hematopoietic progenitor cells in small cell lung cancer patients with favorable prognosis. $J$ Thorac Oncol. 2007;2:51-8.

6. Kalemkerian GP, Akerley W, Bogner P, Borghaei H, Chow L, Downey RJ. et al. Small cell lung cancer. J Natl Compr Canc Netw. 2011;9:1086-113.

7. Che K, Shen H, Qu X, Pang Z, Jiang Y, Liu S. et al. Survival Outcomes for Patients with Surgical and Non-Surgical Treatments in Stages I-III Small-Cell Lung Cancer. J Cancer. 2018;9:1421-9.

8. Counago F, Montemuino S, Martin M, Taboada B, Calvo-Crespo P, Samper-Ots MP. et al. Prognostic factors in neoadjuvant treatment followed by surgery in stage IIIA-N2 non-small cell lung cancer: a multi-institutional study 
by the Oncologic Group for the Study of Lung Cancer (Spanish Radiation Oncology Society). Clin Transl Oncol. 2018.

9. Ning Y, Bao M, Yan X, Xie D, Jiang G. Surgery for advanced non-small cell lung cancer patient after epidermal growth factor receptor tyrosine kinase inhibitor neoadjuvant therapy. Ann Transl Med. 2018;6:407.

10. Schreiner W, Gavrychenkova S, Dudek W, Rieker RJ, Lettmaier S, Fietkau R. et al. Pathologic complete response after induction therapy-the role of surgery in stage IIIA/B locally advanced non-small cell lung cancer. J Thorac Dis. 2018;10:2795-803.

11. Gonzalez-Rivas D, Fieira E, Delgado M, Mendez L, Fernandez R, de la Torre M. Is uniportal thoracoscopic surgery a feasible approach for advanced stages of non-small cell lung cancer? J Thorac Dis. 2014;6:641-8.

12. Lv C, Ma Y, Wu N, Yan S, Zheng Q, Sun Y. et al. A retrospective study: platinum-based induction chemotherapy combined with gemcitabine or paclitaxel for stage IIB-IIIA central non-small-cell lung cancer. World J Surg Oncol. 2013;11:76.

13. Uramoto $H$, Akiyama $H$, Nakajima $Y$, Kinoshita $H$, Inoue $T$, Kurimoto F. et al. The long-term outcomes of induction chemoradiotherapy followed by surgery for locally advanced non-small cell lung cancer. Case Rep Oncol. 2014;7:700-10.

14. Singh AK, Hennon M, Ma SJ, Demmy TL, Picone A, Dexter EU. et al. A pilot study of stereotactic body radiation therapy (SBRT) after surgery for stage III non-small cell lung cancer. BMC Cancer. 2018;18:1183.

15. Scagliotti GV, Pastorino U, Vansteenkiste JF, Spaggiari L, Facciolo F, Orlowski TM. et al. Randomized phase III study of surgery alone or surgery plus preoperative cisplatin and gemcitabine in stages IB to IIIA non-small-cell lung cancer. J Clin Oncol. 2012;30:172-8.

16. Lu T, Yang X, Huang Y, Zhao M, Li M, Ma K. et al. Trends in the incidence, treatment, and survival of patients with lung cancer in the last four decades. Cancer Manag Res. 2019;11:943-53.

17. Kawano D, Okamoto T, Fujishita T, Suzuki Y, Kitahara H, Shimamatsu S. et al. Surgical results of resectable small cell lung cancer. Thorac Cancer. 2015;6:141-5.

18. Friedberg J, Lotano VE. Role of surgery for small cell lung cancer. Curr Probl Cancer. 2012;36:117-30.

19. Shepherd FA, Ginsberg RJ, Haddad R, Feld R, Sagman U, Evans WK. et al. Importance of clinical staging in limited small-cell lung cancer: a valuable system to separate prognostic subgroups. The University of Toronto Lung Oncology Group. J Clin Oncol. 1993;11:1592-7.

20. Szczesny TJ, Szczesna A, Shepherd FA, Ginsberg RJ. Surgical treatment of small cell lung cancer. Semin Oncol. 2003;30:47-56.

21. Schreiber D, Rineer J, Weedon J, Vongtama D, Wortham A, Kim A. et al. Survival outcomes with the use of surgery in limited-stage small cell lung cancer: should its role be re-evaluated? Cancer. 2010;116:1350-7.

22. Weksler B, Nason KS, Shende M, Landreneau RJ, Pennathur A. Surgical resection should be considered for stage I and II small cell carcinoma of the lung. Ann Thorac Surg. 2012;94:889-93.

23. Combs SE, Hancock JG, Boffa DJ, Decker RH, Detterbeck FC, Kim AW. Bolstering the case for lobectomy in stages I, II, and IIIA small-cell lung cancer using the National Cancer Data Base. I Thorac Oncol. 2015;10:316-23.

24. Eberhardt W, Korfee S. New approaches for small-cell lung cancer: local treatments. Cancer Control. 2003;10:289-96.

25. Yang CF, Chan DY, Speicher PJ, Gulack BC, Wang X, Hartwig MG. et al. Role of Adjuvant Therapy in a Population-Based Cohort of Patients With Early-Stage Small-Cell Lung Cancer. J Clin Oncol. 2016;34:1057-64.

26. Brock MV, Hooker CM, Syphard JE, Westra W, Xu L, Alberg AJ. et al. Surgical resection of limited disease small cell lung cancer in the new era of platinum chemotherapy: Its time has come. J Thorac Cardiovasc Surg. 2005;129:64-72.

27. An C, Jing W, Zhang Y, Liu S, Wang H, Zhu K. et al. Thoracic radiation therapy could give survival benefit to elderly patients with extensive-stage small-cell lung cancer. Future Oncol. 2017;13:1149-58.

28. Yang CJ, Chan DY, Shah SA, Yerokun BA, Wang XF, D'Amico TA. et al. Long-term Survival After Surgery Compared With Concurrent Chemoradiation for Node-negative Small Cell Lung Cancer. Ann Surg. 2017.

29. Yao Y, Zhou Y, Yang Z, Huang H, Shen H. Adjuvant chemotherapy following surgical resection improves survival in patients with early-stage small cell lung cancer. Oncol Res. 2018.

30. Takei H, Kondo H, Miyaoka E, Asamura H, Yoshino I, Date H. et al. Surgery for small cell lung cancer: a retrospective analysis of 243 patients from Japanese Lung Cancer Registry in 2004. J Thorac Oncol. 2014;9:1140-5.

31. Rostad H, Naalsund A, Jacobsen R, Strand TE, Scott H, Heyerdahl Strom E. et al. Small cell lung cancer in Norway. Should more patients have been offered surgical therapy? Eur J Cardiothorac Surg. 2004;26:782-6.

32. Tsuchiya R, Suzuki K, Ichinose Y, Watanabe Y, Yasumitsu T, Ishizuka N et al. Phase II trial of postoperative adjuvant cisplatin and etoposide in patients with completely resected stage I-IIIa small cell lung cancer: the Japan Clinical Oncology Lung Cancer Study Group Trial (JCOG9101). J Thorac Cardiovasc Surg. 2005;129:977-83.

33. Shepherd FA, Evans WK, Feld R, Young V, Patterson GA, Ginsberg R. et al. Adjuvant chemotherapy following surgical resection for small-cell carcinoma of the lung. I Clin Oncol. 1988;6:832-8.

34. Eberhardt W, Stamatis G, Stuschke M, Wilke H, Muller MR, Kolks S. et al. Prognostically orientated multimodality treatment including surgery for selected patients of small-cell lung cancer patients stages IB to IIIB: long-term results of a phase II trial. Br J Cancer. 1999;81:1206-12.
35. Janssen-Heijnen ML, Maas HA, Koning CC, van der Bruggen-Bogaarts BA, Groen HJ, Wymenga AN. Tolerance and benefits of treatment for elderly patients with limited small-cell lung cancer. J Geriatr Oncol. 2014;5:71-7.

36. Pallis AG, Shepherd FA, Lacombe D, Gridelli C. Treatment of small-cell lung cancer in elderly patients. Cancer. 2010;116:1192-200.

37. Hu SS, Neff L, Agaku IT, Cox S, Day HR, Holder-Hayes E. et al. Tobacco Product Use Among Adults - United States, 2013-2014. MMWR Morb Mortal Wkly Rep. 2016;65:685-91.

38. Wakeam E, Acuna SA, Leighl NB, Giuliani ME, Finlayson SRG, Varghese TK. et al. Surgery Versus Chemotherapy and Radiotherapy For Early and Locally Advanced Small Cell Lung Cancer: A Propensity-Matched Analysis of Survival. Lung Cancer. 2017;109:78-88. 